American Journal of Applied Sciences 7 (9): 1237-1242, 2010

ISSN 1546-9239

(C) 2010 Science Publications

\title{
Wall Shear Stress Distribution in Patient Specific Coronary Artery Bifurcation
}

\author{
${ }^{1}$ Vahab Dehlaghi and ${ }^{2}$ Frank Gijsen \\ ${ }^{1}$ Department of Biomedical Engineering and Physics, \\ Kermanshah University of Medical Sciences, P.O. Box 1568, Kermanshah, Iran \\ ${ }^{2}$ Department of Biomedical Engineering, Thoraxcenter, \\ Erasmus Medical Center, Rotterdam, The Netherlands
}

\begin{abstract}
Problem statement: Atherogenesis is affected by hemodynamic parameters, such as wall shear stress and wall shear stress spatial gradient. These parameters are largely dependent on the geometry of arterial tree. Arterial bifurcations contain significant flow disturbances. Approach: The effects of branch angle and vessel diameter ratio at the bifurcations on the wall shear stress distribution in the coronary arterial tree based on CT images were studied. CT images were digitally processed to extract geometrical contours representing the coronary vessel walls. The lumen of the coronary arteries of the patients was segmented using the open source software package (VMTK). The resulting lumens of coronary arteries were fed into a commercial mesh generator (GAMBIT, Fluent Inc.) to generate a volume that was filled with tetrahedral elements. The FIDAP software (Fluent Corp.) was used to carry out the simulation by solving Navier-Stokes equations. The FIELDVIEW software (Version 10.0, Intelligent Light, Lyndhurst, NJ) was used for the visualization of flow patterns and the quantification of wall shear stress. Post processing was done with VMTK and MATLAB. A parabolic velocity profile was prescribed at the inlets and outlets, except for 1. Stress free outlet was assigned to the remaining outlet. Results: The results show that for angle lower than $90^{\circ}$, low shear stress regions are observed at the non-flow divider and the apex. For angle larger than $90^{\circ}$, low shear stress regions only at the nonflow divider. By increasing of diameter of side branch ratio, low shear stress regions in the side branch appear at the non-flow divider. Conclusion: It is concluded that not only angle and diameter are important, but also the overall 3D shape of the artery. More research is required to further quantify the effects angle and diameter on shear stress patterns in coronaries.
\end{abstract}

Key words: Wall shear stress, bifurcations, coronary artery, computational fluid dynamics

\section{INTRODUCTION}

Atherogenesis is affected by hemodynamic parameters, such as Wall Shear Stress (WSS), Wall Shear Stress Spatial Gradient (WSSG) and Oscillatory Shear Index (OSI) (Mohammad et al., 2010; Soulis et al., 2006; 2007; Huo et al., 2007; 2009). These parameters are strongly influenced by flow disturbances, which are largely dependent on the geometry of arterial tree (Prosi et al., 2004). The locations with low WSS and high OSI have been thought to be atherosclerotic-prone (Gijsen et al., 2008; Chen et al., 2006; Naderi et al., 2009). The coronary arterial system is an important clinical site of atherosclerosis. The changes in geometry of blood vessels during branching can lead to significant flow disturbances (e.g., flow separation, secondary flow, stagnation point flow, reversed flow and/or turbulence) due to convective inertia (Chen and Lu, 2006).

The Computational Fluid Dynamic (CFD) model has been used to describe the flow patterns in different anatomical geometries. The pulsatile flow field in an anatomically realistic model of the bifurcation of the left anterior Descending Coronary Artery (LAD) and its first Diagonal branch (D1) was simulated numerically and measured by laser Doppler anemometry (Perkthold et al., 1998). The flows in these models exhibited many of the features seen in simpler models, but they also showed that there is considerable individual variability in the flow field in specific regions of the tree and perhaps more important, that there were qualitative differences in some cases between the flow field in idealized and in highly realistic geometries.

Corresponding Author: Vahab Dehlaghi, Department of Biomedical Engineering and physics,

Kermanshah University of Medical Sciences, P.O. Box 1568, Kermanshah, Iran

Tel: (0098-831)-4274618 Fax: (0098-831)- 4276477 
The relationship of vascular geometry and pathophysiology has been the subject of a number of investigations (Caroline et al., 2007; Cheng et al., 2006). These investigations suggest that there are significant relationships between coronary arterial geometry and histomorphometry. Each geometric variable affected the morphometrics differently. With respect to the effect of branch angle, this research has been shown that large angles are associated with intimal thickening and are presumably adverse. Thus, the relation between branch angle and intimal thickness seen here is plausible. The shear stress distribution distal to a branch is not circularly symmetric and it may be significant that the mean intimal thickness of the thickest quadrant of the vessel cross-section correlated with angle more strongly than did the mean intimal thickness of the entire cross-section.

Branch area ratio was primarily correlated with medial variables. The effect of area ratio on medical dimensions decays rapidly, indicating that the medical thickening of the LAD associated with the presence of a branch is localized to its immediate neighborhood.

The identification of relationships between geometric variables and hemodynamic patterns can be used to infer which mechanical factors are more likely to be involved in the localization of disease (Dehlaghi et al., 2007; 2008a; 2008b; Wellnhofer et al., 2009).

Computational Fluid Dynamics (CFD) methods based on in vivo three-dimensional vessel reconstructions have recently been shown to provide prognostically relevant hemodynamic data. With the development of Computed Tomography (CT), MRI angiography and rotational angiography, patient-specific 3D geometries of coronary arteries are readily available for studies of hemodynamics and resulted in a number of recently published researches (Ramcharitar et al., 2008; Gijsen et al., 2008; van der Giessen et al., 2009; Goubergrits et al., 2009; 2008).

A new imaging technique was applied to generate a 3-D reconstruction of a human coronary artery bifurcation in vivo (Gijsen et al., 2007). The observed relationship between SS, WT and remodeling in this specific patient illustrates the spatial heterogeneity of the atherosclerosis in the vicinity of arterial bifurcations. The main geometrical parameters are the angle between the two branches and the surface ratio of the two daughter branches. Some limitations in the current study should be mentioned. The example used in this study was the bifurcation of the LAD with the 2nd diagonal. The angle between the two daughter branches, although being representative for bifurcation angles in the coronary tree, is relatively small. The bifurcation between the LAD and the LCX would pose additional challenges since the lumen of the LCX will disappear more quickly from the IVUS images due to the larger bifurcation angle. The flow division between the LADdist and the 2nd diagonal is controlled by combining stress free outlet boundary conditions with adjustment of the length of the 2nd diagonal to create identical average SS in the proximal part of the two daughter branches. Whether the assumption of equal average SS is valid for mildly diseased coronary arteries is not studied in detail yet.

This investigation presents the effect of branch angle and vessel diameter ratio at the bifurcations on the wall shear stress distribution in the coronary arterial tree.

\section{MATERIALS AND METHODS}

We studied 9 coronary arteries (3 RCA, 2 LCX, 3 LAD and $1 \mathrm{LM}$ ). CT images were digitally processed to extract geometrical contours representing the coronary vessel walls. The lumen of the coronary arteries of the patients was segmented using the open source software package VMTK. With VMTK, we selected trash-holds and combine with marching cube techniques to extract lumen of the artery (Fig. 1).

Filtering was applied to smooth the surface (Fig. 2) and clipping of the inlet and outlets was required. The inlet and outlets were extended by straight tubes for easy implementation of the boundary conditions (Fig. 3).

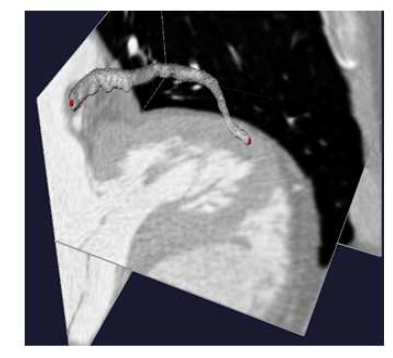

Fig. 1: Extraction of lumen of the artery with VMTK techniques

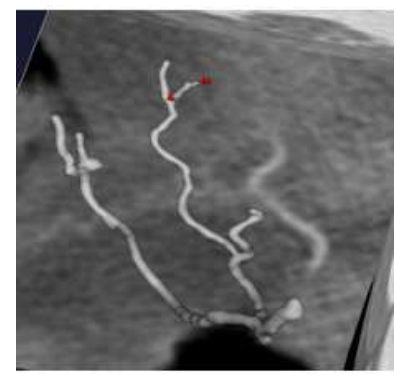

Fig. 2: Smoothing the surface of the artery with VMTK techniques 
The resulting lumens of coronary arteries were fed into a commercial mesh generator (GAMBIT, Fluent Inc.) to generate a volume that was filled with tetrahedral elements. Grid sensitivity tests showed a variation of less than $1 \%$ in the solution of the parameters of interest when the size of volumes decreased $10 \%$. The FIDAP software (Fluent Corp.) was used to carry out the simulation by solving Navier-Stokes equations. The blood flow was modeled as an incompressible Newtonian viscous fluid governed by Navier-Stokes equations. Taking into account the previously mentioned assumptions, the governing equations are:

$\frac{\partial \rho}{\partial t}+\nabla \bullet(\rho U)=0$

$\rho \frac{\mathrm{DU}}{\mathrm{Dt}}=\rho \cdot \mathrm{g}-\nabla \mathrm{P}+\mu \nabla^{2} \mathrm{U}$

Where:

$\rho=$ Fluid density $\left(\mathrm{kg} \mathrm{m}^{-3}\right)$

$\mathrm{U}=$ Velocity vector $\left(\mathrm{m} \mathrm{sec}^{-1}\right)$

$\mathrm{P}=$ Pressure $(\mathrm{Pa})$

$\mathrm{G}=$ Gravity acceleration $\left(\mathrm{m} \mathrm{sec}^{-2}\right)$

$\mu=$ Fluid viscosity (Pa.s)

A parabolic velocity profile was prescribed at the inlets and outlets, except for 1 . Stress free outlet was assigned to the remaining outlet. The dynamic viscosity of blood and its density were assumed to be $3.5 \times 10^{-3}$ Pa.s and $1050 \mathrm{~kg} \mathrm{~m}^{-3}$, respectively (Goubergrits et al., 2009; 2008; Gijsen et al., 2007). No-slip condition was applied at the wall and the wall was assumed to be rigid. The FIELDVIEW software (Version 10.0, Intelligent Light, Lyndhurst, NJ) was used for the visualization of flow patterns and the quantification of wall shear stress. Post processing was done with VMTK and MATLAB.

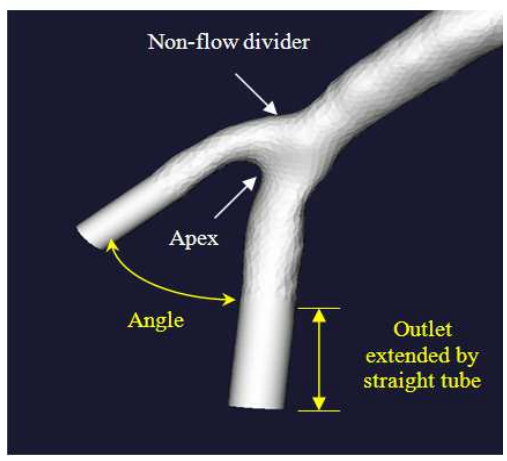

Fig. 3: Extension the inlet and outlets by straight tubes

\section{RESULTS AND DISCUSSION}

Steady blood flow in the 9 reconstructed models of the coronary artery was simulated using the CFD program FIDAP.

Influence of angle of side branch at the bifurcations on the WSS distribution: Figure 4 shows the wall shear stress distribution in the Right Coronary Artery (RCA). In this case angle of side branch at the bifurcation is larger than $90^{\circ}$. Low shear stress regions present at non-flow divider. Absolute values of WSS at the non-flow divider are smaller than $0.28(\mathrm{~Pa})$. Opposite the non-flow divider, near the apex a small region with low shear stress is present (Fig. 4). Absolute values of WSS at this region are larger than $0.4(\mathrm{~Pa})$. For angle larger than $90^{\circ}$, low shear stress regions only at the nonflow divider.

In the 2 RCA models that the angle of side branch at the bifurcation was larger than $90^{\circ}$, we observed that low shear stress region present only at the non-flow divider area. Absolute values of WSS at the non-flow divider varied between 0.26-0.35 (Pa). In the apex region this value is larger than $0.5(\mathrm{~Pa})$ (Table 1).

Table 1:Absolute values of WSS at the non-flow divider and apex for different angle of side branch at the bifurcation

\begin{tabular}{lccc}
\hline & & $\begin{array}{l}\text { Apex } \\
\text { WSS }\end{array}$ & $\begin{array}{l}\text { Non-flow divider } \\
\text { WSS }\end{array}$ \\
\hline RCA-59 MB-SB1 & $>90$ & 0.40 & 0.28 \\
RCA-59 MB-SB2 & $>90$ & $>0.50$ & 0.35 \\
RCA-89 MB-SB2 & $>90$ & $>0.50$ & 0.26 \\
RCA-44 MB-SB1 & 90 & $>0.50$ & 0.33 \\
RCA-89 MB-SB1 & $<90$ & 0.26 & 0.36 \\
LAD-72 MB-SB1 & $<90$ & 0.26 & $>0.50$ \\
LCX-59 MB-SB2 & $<90$ & 0.37 & 0.34 \\
\hline
\end{tabular}

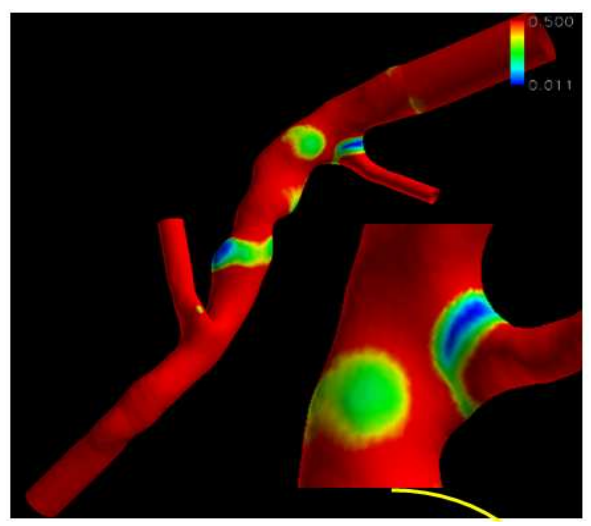

Fig. 4: WSS distribution in the right coronary artery with side branch angle larger than $90^{\circ}$ 


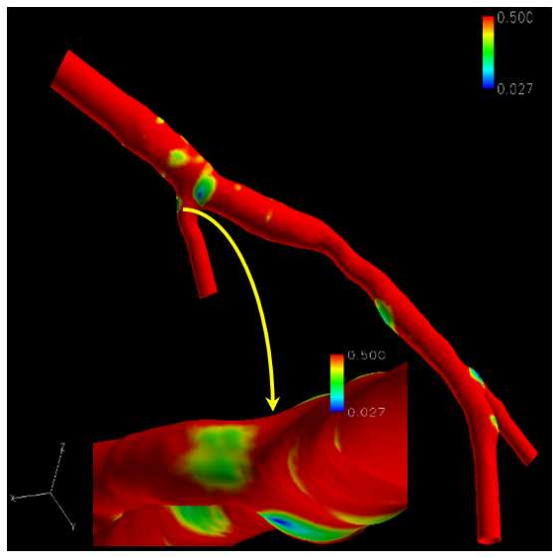

Fig. 5: WSS distribution in the LAD coronary artery with side branch angle smaller than $90^{\circ}$

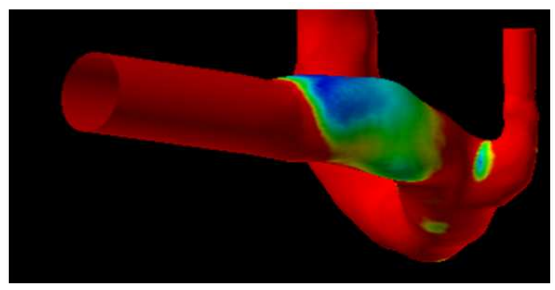

Fig. 6: WSS distribution in the right coronary artery with side branch angle approximately $90^{\circ}$

Table 2: Absolute values of WSS at the non-flow divider and apex for different diameter of side branch at the bifurcation

\begin{tabular}{llll}
\hline & & Apex & Non-flow divider \\
& SD/MD & WSS & WSS \\
\hline LAD-72 MB-SB1 & Small & $>0.5$ & $>0.50$ \\
LAD-59 MB-SB1 & Large & $>0.5$ & 0.40 \\
LAD-72 MB-SB2 & Small & $>0.5$ & $0.4,>0.5$ \\
LAD-59 MB-SB2 & Large & $>0.5$ & 0.25 \\
LCX-72 MB-SB2 & Small & $>0.5$ & $>0.50$ \\
LCX-59 MB-SB2 & Large & $>0.5$ & 0.30 \\
\hline
\end{tabular}

Figure 5 illustrates the wall shear stress distribution in the left anterior descending of coronary artery (LAD). In this case angle of side branch at the bifurcation is smaller than $90^{\circ}$. The results show that for angle lower than $90^{\circ}$, low shear stress regions are observed at the non-flow divider and the apex. Absolute values of WSS at the non-flow divider varied between $0.36-0.5(\mathrm{~Pa})$. In the apex region this value is varied between $0.26-0.37(\mathrm{~Pa})$.

When the angle of the side branch at the bifurcation is approximately $90^{\circ}$ (Fig. 6), we observed that a small region of low shear stress present only at the non-flow divider area. Absolute value of WSS at the non-flow divider is $0.33(\mathrm{~Pa})$. In the apex region this value is larger than $0.5(\mathrm{~Pa})$ (Table 1).

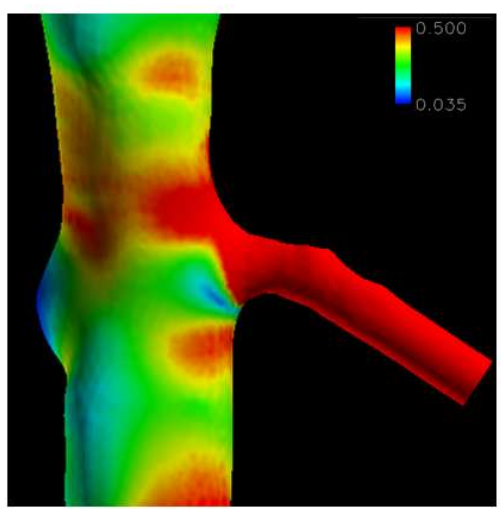

Fig. 7: WSS distribution in the LAD coronary artery with small side branch

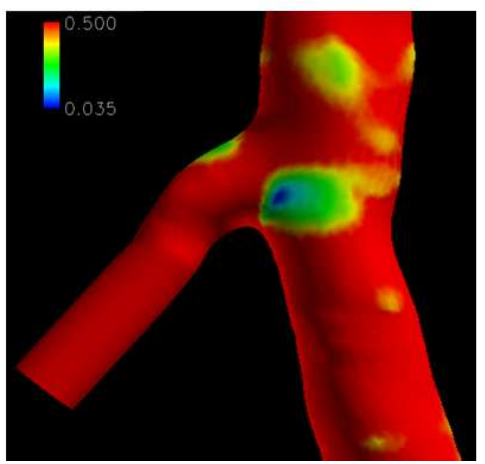

Fig. 8: WSS distribution in the LAD coronary artery with large side branch

Influence of diameter of side branch at the bifurcations on the WSS distribution: WSS distribution in the LAD coronary artery with small side branch was shown in Fig. 7. In this case shear stress in the side branch is larger than $0.5 \mathrm{~Pa}$, both at the apex and the non-flow divider.

WSS distribution in the LAD coronary artery with larger side branch was shown in Fig. 8. In this case low shear stress regions in the side branch appear at the non-flow divider. Absolute values of WSS at the nonflow divider varied between 0.25-0.4 (Pa). In the apex region this value is larger than $0.5(\mathrm{~Pa})$ (Table 2).

\section{CONCLUSION}

If the angle is lower than $90^{\circ}$, low shear stress regions are observed at the non-flow divider and the apex. For angle larger than $90^{\circ}$, low shear stress regions only at the non-flow divider and shear stress at the nonflow divider seem to be lower than bifurcation with angle smaller than $90^{\circ}$. 
By increasing the ratio of side branch diameter to main branch diameter (Ds/Dm), low shear stress regions in the side branch appear at the non-flow divider

We concluded that not only angle and diameter, but also the overall 3D shape of the artery are important. More research is required to further quantify the effects angle and diameter on shear stress patterns in coronaries.

The current results confirm and extend our previous observations and further demonstrate the importance of artery geometry on the intravascular fluid dynamics.

\section{ACKNOWLEDGEMENT}

The researchers thank J.J. Wentzel, Alina G. van der Giessen and (Department of Biomedical Engineering, Thoraxcenter, Erasmus Medical Center, Rotterdam) for their technical and computational support.

\section{REFERENCES}

Caroline, C., F. Helderman, D. Tempel, D. Segers and B. Hierck et al., 2007. Large variations in absolute wall shear stress levels within one species and between species. Atherosclerosis, 195: 225-235. DOI: 10.1016/j.atherosclerosis.2006.11.019

Cheng, C., D. Tempel, R. van Haperen A. van der Baan and F. Grosveld et al., 2006. Atherosclerotic lesion size and vulnerability are determined by patterns of fluid shear stress. Circulation, 113: 2744-2753. PMID: 16754802

Chen, J. and X.Y. Lu, 2006. Numerical investigation of the non-Newtonian blood flow in a bifurcation model with a non-planar branch. J. Biomech., 39, 818-832. DOI: 10.1016/j.jbiomech.2005.02.003

Chen, J., X.Y. Lu and W. Wang, 2006. Non-Newtonian effects of blood flow on hemodynamics in distal vascular graft anastomoses. J. Biomech., 39: 1983-1995. DOI: 10.1016/j.jbiomech.2005.06.012.

Dehlaghi, V., M.T. Shadpour and S. Najarian, 2007. Numerical analysis of pulsatile blood flow in a stented human coronary artery with a flow divider. Am. J. Applied Sci., 4: 397-404. http://www.scipub.org/fulltext/ajas/ajas46397-404.pdf

Dehlaghi, V., S. Najarian and M.T. Shadpour, 2008a. Effect of stent geometry on phase shift between pressure and flow waveforms in stented human coronary artery. Am. J. Applied Sci., 5: 340-346. http://www.scipub.org/fulltext/ajas/ajas54340-346.pdf
Dehlaghi, V., M.T. Shadpour and S. Najarian, 2008b. Analysis of wall shear stress in stented coronary artery using 3D computational fluid dynamics modeling. J. Mater. Process. Technol., 197: 174-181. DOI: 10.1016/j.jmatprotec.2007.06.010

Gijsen, F.J.H., J.J. Wentzel, A. Thury, B. Lamersa and J.C.H. Schuurbiers et al., 2007. A new imaging technique to study 3-D plaque and shear stress distribution in human coronary artery bifurcations in vivo. J. Biomech., 40: 2349-2357. DOI: 10.1016/j.jbiomech.2006.12.007

Gijsen, F.J.H., J.J. Wentzel, A. Thury, F. Mastik and J.A. Schaar et al., 2008. Strain distribution over plaques in human coronary arteries relates to shear stress. Am. J. Physiol. Heart Circ. Physiol., 295: H1608-H1614. DOI: 10.1152/ajpheart.01081.2007

Goubergrits, L., U. Kertzscher, B. Schoneberg, E. Wellnhofer and C. Petz et al., 2008. CFD analysis in an anatomically realistic coronary artery model based on non-invasive 3D Imaging: Comparison of magnetic resonance imaging with computed tomography. Int. J. Cardiovasc. Imag., 24: 411-421. DOI: $10.1007 / \mathrm{s} 10554-007-9275-\mathrm{z}$

Goubergrits, L., E. Wellnhofer, U. Kertzscher, K. Affeld and C. Petz et al., 2009. Coronary artery WSS profiling using a geometry reconstruction based on biplane angiography. Ann. Biomed. Eng., 37: 682-691. DOI: 10.1007/s10439-009-9656-7

Huo, Y., T. Wischgoll and G.S. Kassab, 2007. Flow patterns in three-dimensional porcine epicardial coronary arterial tree. Am. J. Physiol. Heart Circ. Physiol., 293: H2959-H2970. DOI: 10.1152/ajpheart.00586.2007

Huo, Y., J.S. Choy, M. Svendsen, A.K. Sinha and G.S. Kassab, 2009. Effects of vessel compliance on flow pattern in porcine epicardial right coronary arterial tree. J. Biomech., 42: 594-602. DOI: 10.1016/j.jbiomech.2008.12.011

Mohammad, F.I., S. Awawdeh, A. Saleh and N.A. Bashir, 2010. Total level of serum homocysteine in males and females with coronary heart disease of different age groups. Am. J. Biochem. Biotechnol., 6: 116-119. http://www.scipub.org/fulltext/ajbb/ajbb62116-119.pdf

Naderi, F., A. Heideri, M. Kargarzadeh and M. Tahmasbi, 2009. The effects of open heart surgery (coronary bypass) on depression and social adjustment of hospitalized heart patients. J. Soc. Sci., 5: 304-313. http://www.scipub.org/fulltext/jss/jss54304-313.pdf

Perkthold, K., M. Hofer, G. Rappitsch, M. Loew and B.D. Kuban et al., 1998. Validated computation of physiologic flow in a realistic coronary artery branch. J. Biomech., 31: 217-228. PMID: 9645536 
Prosi, M., K. Perkthold, Z. Ding and M.H. Friedman, 2004. Influence of curvature daynamics on pulsatile coronary artery flow in a realistic bifurcation model. J. Biomech., 37: 1767-1775. DOI: 10.1016/j.jbiomech.2004.01.021

Ramcharitar, S., J. Daeman, M. Patterson, R.J. van Guens and E. Boersma et al., 2008. First direct in vivo comparison of two commercially available threedimensional quantitative coronary angiography systems. Catheter. Cardiovasc. Interv., 71: 44-50. PMID: 18098181

Soulis, J.V., T.M. Farmakis, G.D. Giannoglou and G.E. Louridas, 2006. Wall shear stress in normal left coronary artery tree. J. Biomech., 39: 742-749. DOI: 10.1016/j.jbiomech.2004.12.026

Soulis, J.V., G.D. Giannoglou, G.E. Parcharidis and G.E. Louridas, 2007. Flow parameters in normal left coronary artery tree. Implication to Atherogenesis. Comput. Biol. Med., 37: 628-636. DOI: 10.1016/j.compbiomed.2006.06.006
Van der Giessen, A.G., J.J. Wentzel, W.B. Meijboom, N.R. Mollet and A.F. van der Steen et al., 2009. Plaque and shear stress distribution in human coronary bifurcations: A multislice computed tomography study. Eurointervention, 4: 654-661. PMID: 19378688

Wellnhofer, E., L. Goubergrits, U. Kertzscherm, K. Affeld and E. Fleck, 2009. Novel non-dimensional approach to comparison of wall shear stress distributions in coronary arteries of different groups of patients. Atherosclerosis, 202: 483-490. DOI: 10.1016/j.atherosclerosis.2008.05.044 